\title{
Folded chaotic whispering-gallery modes in non-convex, waveguide-coupled planar optical microresonators
}

\author{
Kahli Burke and Jens U. Nöckel* \\ Department of Physics \\ University of Oregon
}

(Dated: December 23, 2019)

\begin{abstract}
Chaotic whispering-gallery modes have significance both for optical applications and for our understanding of the interplay between wave phenomena and the classical ray limit in the presence of chaotic dynamics and openness. In strongly non-convex geometries, a theorem by Mather rules out the existence of invariant curves in phase space corresponding to rays circulating in whispering-gallery patterns, so that no corresponding modes of this type are expected. Here we discuss numerical computations of the electromagnetic fields in planar dielectric cavities that are strongly non-convex because they are coupled to waveguides. We find a family of special states which retains many features of the chaotic whispering-gallery modes known from convex shapes: an intensity pattern corresponding to near-grazing incidence along extended parts of the boundary, and comparatively high cavity $\mathrm{Q}$ factors. The modes are folded into a figure-eight pattern, so overlap with the boundary is reduced in the region of self-intersection. The modes combine the phenomenology of chaotic whispering-gallery modes with an important technological advantage: the ability to directly attach waveguides without spoiling the Q factor of the folded mode. Using both a boundary-integral method and the finite-difference time domain technique, we explore the dependence of the phenomenon on wavelength in relation to cavity size, refractive-index contrast to the surrounding medium, and the degree of shape deformation. A novel feature that distinguishes folded from regular whispering gallery modes is that a given shape will support high-Q folded chaotic whispering gallery modes only in certain wavelength windows.
\end{abstract}

\footnotetext{
* To whom correspondence should be addressed: noeckel@uoregon.edu
} 


\section{INTRODUCTION}

Wave-chaotic optical cavities are of interest in applications because of the degrees of design freedom that are opened up when the geometric constraints of separability and symmetry are removed [1]: spectral density, lifetimes and emission directionality can all be tailored in sometimes dramatic ways that go far beyond perturbative effects around separable special cases. Among the first examples are planar dielectric ovals that support chaotic whispering-gallery modes (WGMs) [2], where the anisotropic shape gives rise to highly universal directional emission patterns that can be explained based on the classical phase-space structure of the ray dynamics. From a theoretical point of view, this connection to classical ray physics is intriguing because the transition to chaos that occurs in generic nonseparable systems must then be studied in the presence of openness. The wave equation in this scenario exhibits quasibound states at complex wavenumber $k=k_{\mathrm{r}}-i \kappa$; here $\kappa$ measures the escape rate which is related to the quality factor via

$$
Q=\frac{k}{2 \kappa} \text {. }
$$

WGMs in oval resonators are special states that preserve long lifetimes comparable to those found in circular dielectrics [3], due to the fact that they are predominantly localized in the phase-space region corresponding to total internal reflection at the dielectric boundary. When the invariant curves (tori) that foliate this phase-space region in the limit of circular symmetry are gradually broken up following the KAM theorem as applied to convex billiards [4], regular WGMs become chaotic WGMs in which rays explore regions of phase space in which the rays cease to be confined by total internal reflection. The critical angle for total internal reflection defines an escape window in phase space rather than real space, because this angle is directly related to the tangential momentum component at the surface of the resonator. In Ref. [2], an approximate semiclassical quantization of these unconventional modes was proposed based on a separation of time scales between the fast whisperinggallery circulation and a slower spiraling-in toward the escape window, making it possible to identify adiabatic invariant curves. Their location then served as an initial condition for ray simulations of the escape directionality, caused by the mixed phase space in the vicinity of the escape window.

It has long been understood that no true WGMs can be sustained in circular domains if waveguides are attached, because this creates openings in the boundary that interrupt the 
whispering-gallery circulation. In phase space, the escape window then depends not only on tangential momentum but on position. For a detailed study of this breakdown in the context of electronic microstructures with otherwise impenetrable walls, see Ref. [5]. This can be viewed as a special case of a theorem by Mather [6], who proved the non-existence of whispering-gallery invariant curves in planar billiards when the boundary is not everywhere convex. An example for Mather's theorem is the Bunimovich stadium (two semicircles joined by straight, parallel sides) [7], whose ray dynamics does not permit whispering-gallery orbits even if $\ell$ is arbitrarily short, even though the weaker condition of non-concavity still holds. Strict convexity is therefore required in order to sustain WG circulation, but any waveguide openings will necessarily introduce corners where this condition breaks down.

By exploiting the preferential emission directions of chaotic WGMs in the near or far field, light can be coupled into and out of the resonator without contacting (and thereby perturbing) the boundary geometry directly. However, monolithically attached waveguides offer some distinct advantages from an engineering point of view. In laser applications, they allow both efficient optical pumping and more complete collection of the emission. Although directional emission patterns from planar cavities can be made highly directional in the plane [8], losses by out-of-plane diffraction can be significant [9-12]. With attached waveguides, such losses are minimized. Moreover, waveguides afford precise control over the number of input/output channels.

This has motivated several recent proposals to integrate chaotic cavities with waveguides [13-18]. Because of Mather's theorem, however, almost all such proposals involve modes that are not of whispering-gallery type, achieving good confinement instead with states localized on periodic orbits: Stable and unstable periodic ray orbits whose reflections occur far enough away from any waveguide openings will be insensitive to the boundary shape at the waveguide apertures. These periodic orbits exist with or without the presence of attached waveguides, and they make lasing possible even in the free-standing Bunimovich stadium [19].

The appearance of wavefunction scarring due to unstable periodic orbits in [19] illustrates that wave solutions of the Helmholtz equation can defy ray-optics predictions. This is also true for the predictions of Mather's theorem, and again the stadium billiard is a case in point: Numerical solutions for closed (hard-wall) cavities [20] show that a form of chaotic WGM exists if the straight sides are sufficiently short. 


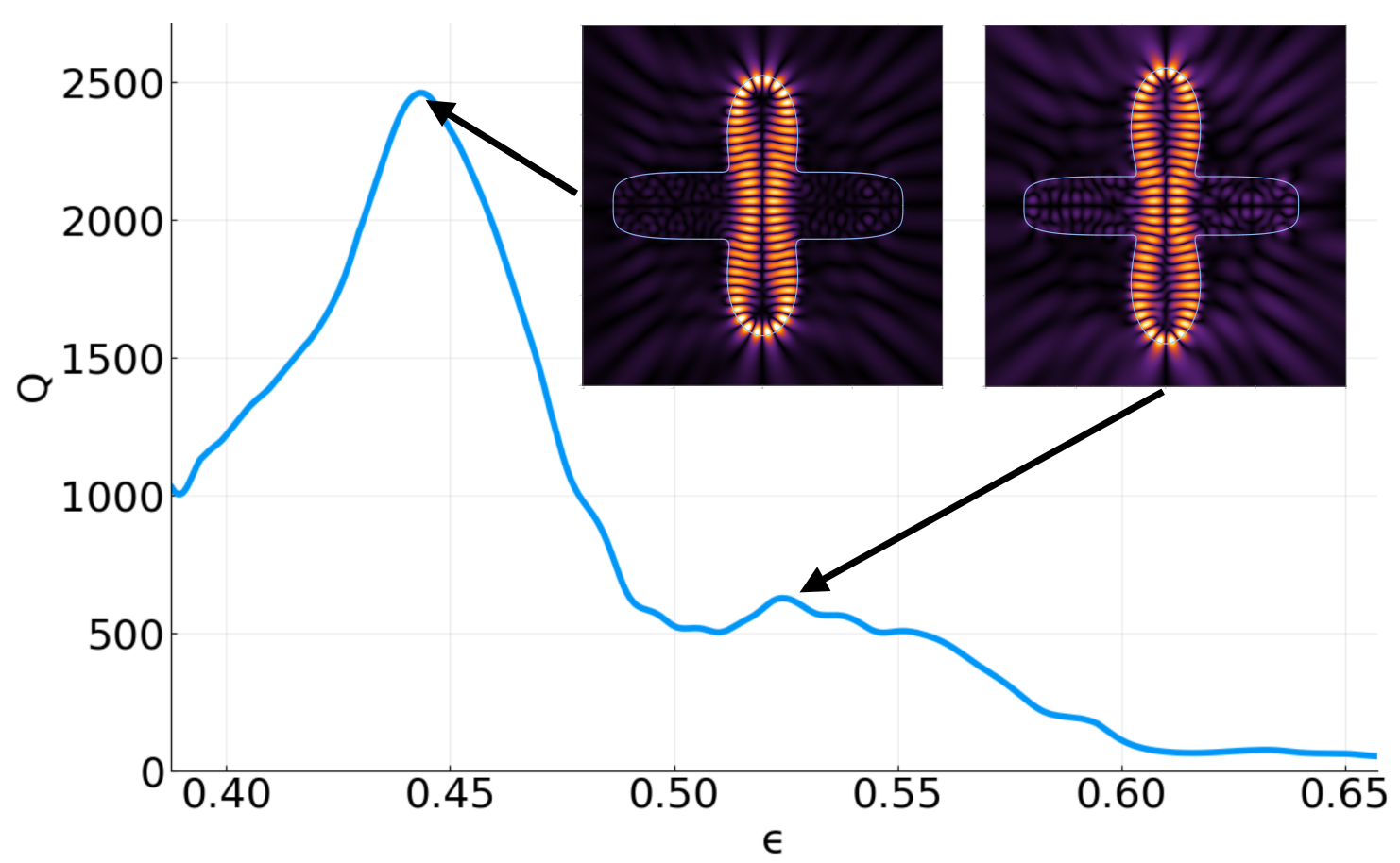

Figure 1. Spatial structure of a folded chaotic whispering-gallery mode (insets), and Q factor of the mode versus deformation parameter $\epsilon$ at refractive index $n=2.4$. The resonator deformation is defined in Eq. (2); attached waveguides of finite lengths are shown horizontal; their lengths are slightly unequal to remove reflection symmetry. False color represents field intensity. The real part of $k$ (not shown) decreases approximately linearly from 14 to 12.5 as $\epsilon$ increases.

We will describe whispering-gallery type behavior with a different topology, which can be described as folded chaotic whispering-gallery modes, cf. Fig. 1. This refers to the fact that the direction of whispering-gallery ray circulation underlying the modes reverses due to a self-intersection near the center of the cavity. The bowtie orbit of Ref. [21] also exhibits a self-intersection, but it - like all other such orbits we are aware of - gives rise to modes that explore the boundary only at isolated points corresponding to the discrete vertices where ray reflections occur. In Ref. [21], $Q>1000$ was obtained at relatively high refractive index contrast of $n \gtrsim 3$, corresponding to a critical angle for total internal reflection of $\chi_{\mathrm{c}} \approx \arcsin 1 / n \approx 0.34$ (where $\chi$ denotes the angle of incidence with respect to the surface normal). For such high index contrast, even Fabry-Perot cavities with near-normal angle of incidence provide similar Q-factors without the need for additional mirrors.

By contrast, all the high-Q modes in our design are localized predominantly in the whispering-gallery region of phase space, much further from the critical angle, even when 
the index contrast is below $n=2$. The light is then well-confined by total internal reflection. Reminiscent of a figure-eight shape, folded chaotic WGMs have a waist that allows them to avoid two isolated sections of the boundary; but unlike stable periodic orbits such as the bowtie, folded chaotic WGMs explore the remainder of the perimeter in the same way as would be expected for conventional WGMs. In this sense, these types of modes come as close as possible to the circulating ray patterns that are strictly ruled out by Mather's theorem.

The self-intersecting topology makes folded chaotic WGMs amenable to the incorporation of waveguides because the openings have only small overlap with the waist of the mode. Although the field looks similar to Gaussian beam in the waist region, the latter are fundamentally different because they are always built upon stable periodic orbits, which in the simplest case requires a configuration corresponding to two focusing mirrors [22]. In our design, the reflections are near-grazing along the entire convex part of the boundary, and there is no focusing-mirror configuration. Therefore, folded chaotic WGMs cannot be obtained in paraxial optics.

As an important correction to the ray limit in the presence of chaotic dynamics, $d y$ namical localization has been invoked [2] to explain the high Q factors of chaotic WGMs in convex cavities, and circular dielectrics with corrugated surface perturbations [23]. A hallmark of dynamical localization is an exponential decay of wave intensity as a function of a variable which in the classical ray picture exhibits diffusive time evolution. In corrugated perturbed circles, this variable is the angular momentum; and it remains a good quantity to characterize dynamical localization in chaotic WGMs of smooth but convex cavities [24]. At each reflection, the angular momentum is proportional to $\sin \chi$. However, an important common feature on which previous work relies is that the underlying classical dynamics exhibits a separation of time scales, without which diffusive behavior cannot be identified. Before $\sin \chi$ changes significantly, a whispering-gallery ray will have completed many round trips, allowing the azimuthal positions of boundary reflections to be averaged out [24].

To identify wave localization in the folded WGMs to be discussed here, we have to follow a different route because angular momentum does not undergo the required slow diffusion. It jumps discontinuously because a figure-eight pattern entails periodic reversals of the sense of rotation. Therefore, we begin with a description of the ray dynamics in phase space before presenting the detailed results of our wave calculations in section III. These two descriptions are then synthesized in section IV to identify the classical structures on which the modes 

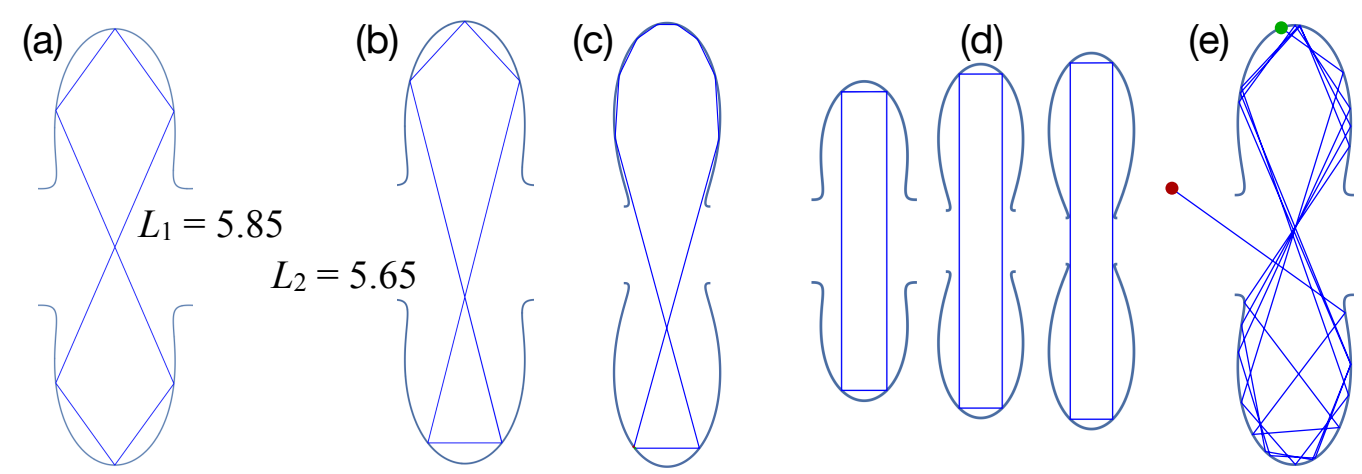

Figure 2. (a-c): Three self-intersecting unstable periodic orbits with angles of incidence $\sin \chi \geq 0.6$ in the billiard shape given by Eq. (2) with $\epsilon=0.43$. The lengths $L_{1}, L_{2}$ of the orbits are slightly different. The same topology of orbits persists for a wide range of deformations. (c) is obtained from (b) by adjusting the initial conditions to generate eight instead of three reflections in the top half. For the rectangular unstable periodic orbit in (d), the increasing proximity to the corners of the waveguide aperture is illustrated for increasing $\epsilon$. (e) A ray trajectory launched in the cavity (green dot) and escaping through the waveguide opening (red dot). It retains the folded character of the orbits in (a), (b) for a short time.

are localized, using Husimi projections of the numerical wave functions onto the ray phase space.

\section{CAVITY SHAPE AND RAY PHASE SPACE}

Figure 2 illustrates the non-convex cavity shape in which the long-lived modes are confined. The openings in the boundary are attached to waveguides, but for the purposes of the ray dynamics they constitute escape windows, in addition to the refractive escape mechanism that sets in when the condition for total internal reflection at the dielectric interface is violated. The three self-intersecting periodic orbits shown in Fig. $2(\mathrm{a}-\mathrm{c})$ have incident angles satisfying $\sin \chi \geq 0.6$, which means they are confined by total internal reflection for refractive indices $n>1.7$. Half of the reflections occur with opposite sense of circulation, corresponding to the reversal of angular momentum (with respect to the center) taking place at the self-intersection.

The geometry also permits a rectangular periodic orbit whose angle of incidence has 
the fixed value $\sin \chi \approx 0.707$, but Fig. $2(\mathrm{~d})$ shows an important distinction to the selfintersecting orbits: as $\epsilon$ increases, the corners of the waveguide aperture encroach on the orbit and eventually touch it. The resulting corner diffraction [25] will degrade the lifetime of any modes based on this orbit. The numerical computations to be described in section III have revealed high-Q modes in cavities of the shape (2), that do show enhanced intensity near the rectangle orbit, but never exclusively on that orbit. Instead, the folded chaotic states consistently show high intensity overlapping with the folded orbits over a wide range of deformations $\epsilon$ and attached waveguide widths.

Figure 2 (e) illustrates a ray in the chaotic interior region that escapes into the waveguide opening after several reflections. By folding a WGM over itself and attaching waveguides in the avoided regions of the boundary, two distinct escape windows in phase space are created: one bounded by the critical angle for refractive escape, and the other by the realspace locations of the waveguide openings. This is illustrated in the Poincaré surface of section of Fig. 3, depicting the phase space of a billiard parametrized in polar coordinates by

$$
r(\phi) \propto 1+\epsilon \cos (4 \phi)
$$

The curve parameter $\phi$ is recorded on the horizontal axis, and $\sin \chi$ is a measure of the conjugate momentum variable.

For the numerical wave calculations of Fig. 1, waveguide segments were connected to the open sections of the boundaries depicted in Fig. 3. The waveguide segments were modeled as a "squircle" [26]. This curve has a polar-coordinate representation

$$
r_{\mathrm{WG}}(\phi)=\sqrt{a^{2}|\cos \phi|+b^{2}|\sin \phi|}
$$

but was also shifted horizontally in order to connect smoothly with the unshifted curve of Eq. (2).

The waveguide portions of the boundary are shaded in the Poincaré section because they are irrelevant to the interior ray dynamics that makes the folded orbits possible. There are no stable islands associated with any periodic orbits other than the bouncing-ball trajectories (leftmost inset) whose perpendicular angle of incidence precludes long-lived stable modes from forming. There are no invariant curves in the whispering-gallery region near $\sin \chi \rightarrow 1$. 


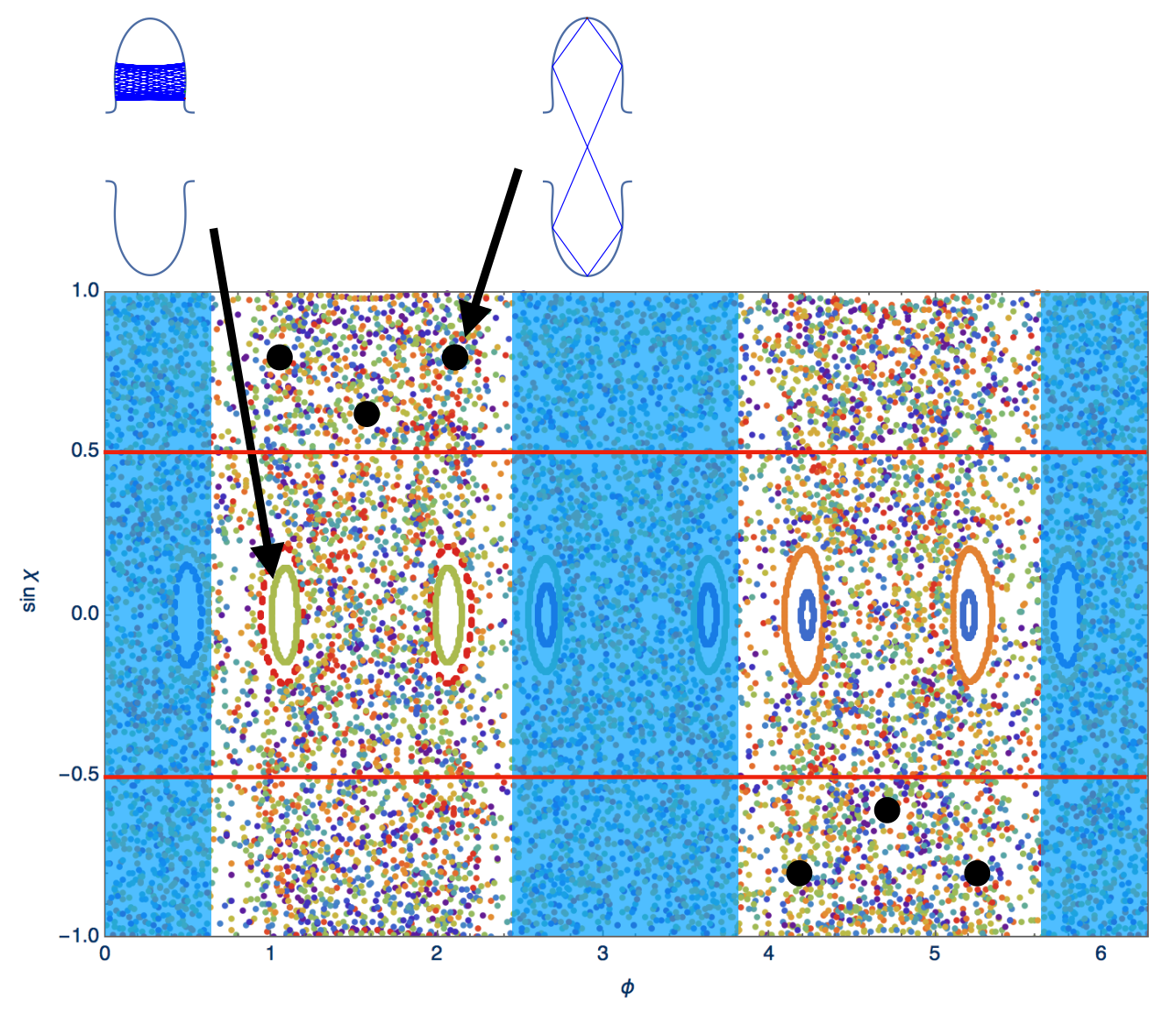

Figure 3. Poincaré surface of section with the curve parameter $\phi$ as position and $\sin \chi$ as momentum variable. Here, $\chi$ is the angle between incident rays and the surface normal, so that $\sin \chi=1$ corresponds to grazing incidence. Ray trajectories show up as point clouds in the chaotic sea, or as one-dimensional lines for regular motion. Shown as insets are a stable bouncing-ball orbit (left) and an unstable periodic orbit (right). Arrows point to the corresponding phase space locations; in particular the 6 black dots indicate the location of the 6 bounce points for the periodic orbit. Shaded boxes indicate the intervals of $\phi$ which describe the attached waveguides. Rays entering these regions will escape the resonator. The thin horizontal lines mark the critical angle for total internal reflection, $\left|\sin \chi_{c}\right|=1 / n(n=2$ here $)$. Here and in all subsequent results, $n$ can be viewed as the interior refractive index while the exterior refractive index is unity.

\section{WAVE CALCULATIONS}

The wave patterns in Fig. 1 indicate that the mode is not supported by any one of the self-intersecting periodic orbits in Fig. 2 alone, but by a more extended phase-space region in their vicinity. The main panel of Fig. 1 follows a single mode over a range of 
deformations $\epsilon$, revealing an optimal deformation of $\epsilon \approx 0.44$ at which this particular mode reaches $Q \approx 2500$.

To characterize the parameter-dependence of the Q factor further, we carry out numerical simulations of the wave equation using two different methods. The first approach is a version of the boundary-integral method described by Heider [27] in which we directly search for the quasibound states satisfying outgoing-wave boundary conditions at infinity [28]. The second approach isolates the high-Q modes by harmonic inversion [29] of temporal field variation collected in a finite-difference time domain simulation with perfectly matched layer boundary condition (MEEP with post-processing by Harminv) [30]. In the direct quasibound-state calculation, we obtain complex wavenumbers $k_{\mathrm{QB}}=k-i \kappa$ to determine Q using Eq. (1).

\section{A. Quasibound states}

In the boundary-integral approach, we leverage the fact that the dielectric defining the billiard is uniform so that a Green-function description of the interior and exterior fields purely in terms of the boundary is possible. At the dielectric interface, the electric field is assumed to satisfy the boundary conditions for TM polarization (electric field perpendicular to the plane). After discretization along the interface, this leads to a nonlinear eigenvalue problem $A\left(k_{\mathrm{QB}}\right) \boldsymbol{u}=\mathbf{0}$ where $\boldsymbol{u}$ contains the source values of the electric field and its normal derivative, and $A$ is a matrix obtained from the field matching equations. A non-trivial solution requires searching for $k_{\mathrm{QB}}$ in the complex plane, which we do using a predictorcorrector method [27]. The method requires discretization of the boundary curve, and we generally found good convergence up to wavenumbers of $k \approx 60$ by choosing 580 points. For additional analysis of the exponential convergence of the method with discretization density, see Ref. [27].

By definition, the boundary-integral method assumes that regions of uniform dielectric constant are bounded by closed curves, so we model the waveguides as finite-length attachments, see Fig. 1. By varying the lengths of these waveguides (either together or independently of each other) we verified that the finiteness of the stubs has no significant effect on the mode structure: neither real parts nor imaginary parts of $k_{\mathrm{QB}}$ for the modes with figureeight topology were affected unless their Q factor was below approximately 200 to begin with. Because the modes are built on classical ray orbits, they will not be found (with appreciable 

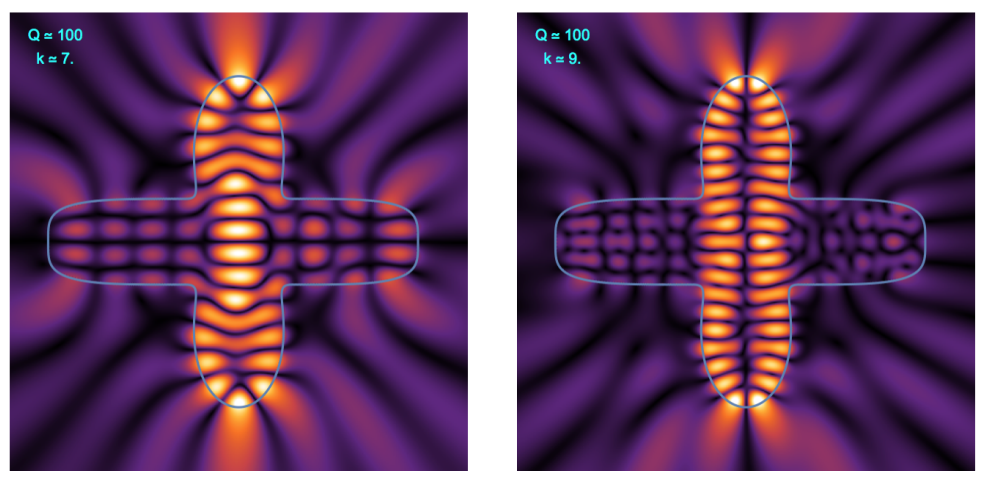

Figure 4. Small-cavity limit of the folded chaotic WGM, showing only low Q factors as the transverse width of the mode is comparable to the cavity size. Below $k \approx 7$, the wavelength is too long to observe the WGM like concentration of intensity near the top and bottom of the cavity. The horizontal waveguide stubs are attached in a way that smoothly matches the shape given by Eq. (2) for the vertical lobes. The refractive index is $n=2.4$ inside and $n=1$ outside, and the vertical lobes are described by Eq. (2) with $\epsilon=0.444$. In this and the following plots, one can discern even and odd parity with respect to reflections at the horizontal axis. Although we intentionally break reflection symmetry across the vertical axis by making the horizontal waveguide lengths unequal, the modes still show approximate antinodes (left) or nodal lines (right) along the vertical axis. This is because the cavity supporting most of the intensity is still left-right symmetric.

lifetimes) in small cavities. This is illustrated in Fig. 4 where the self-intersecting topology is barely discernible in the wave intensities. This raises the question whether the $\mathrm{Q}$ factors of such modes will increase monotonically with increasing cavity size, or equivalently with shorter wave length. To address this dependence, we fix the cavity geometry at $\epsilon=0.444$ to identify all the modes with lifetimes above a threshold of $\left|\operatorname{Im}\left(k_{\mathrm{QB}}\right)\right|=\kappa<0.05$ with wavenumber $k \lesssim 60$. The particular choice of deformation corresponds to the maximum in Fig. 1 at $n=2.4$, but the same phenomena are observed at other values of $\epsilon$.

Figure 5 shows that the $\mathrm{Q}$ factors display a non-monotonic peak structure. The data are plotted for four different resonators with refractive indices of $n=1.6,1.8,2.0$ and 2.4. The peaks are more pronounced for larger refractive index because the critical angle for total internal reflection decreases with $\sin \chi_{\mathrm{c}}=1 / n$. By choosing the horizontal axis to display $n k$ instead of the free-space wavenumber $k$, the peak positions for different $n$ moreover line up to a good approximation. Since $n k$ is the wave number inside the cavity, this indicates that the high-Q peak locations are determined by the interior wave patterns, not the monotonically 


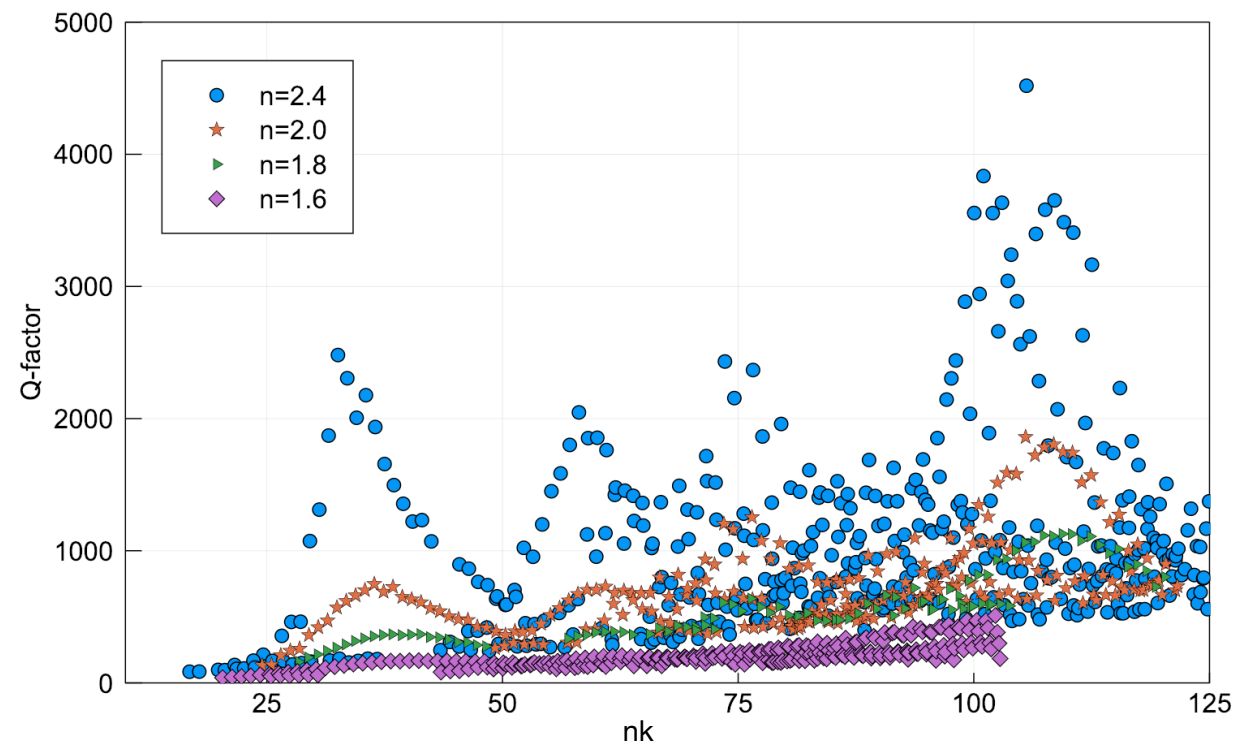

Figure 5. Wavenumber sweeps of the $\mathrm{Q}$ factor for different refractive indices $n$, discarding low$\mathrm{Q}$ modes with $\kappa \geq 0.05$ (for $n=1.6$, only modes with $\kappa \geq 0.2$ are shown). The deformation is $\epsilon=0.444$. The appearance of distinct Q-factor peaks as a function of quasi-bound state wavenumber is most pronounced at the largest refractive index, $n=2.4$, and becomes nearly unobservable at $n=1.6$ (therefore, data for $n=1.6$ were not collected beyond $n k \approx 106.5$ ). The peaks that do remain observable are approximately at the same values of $n k$ for all $n$.

n-dependent coupling to the surrounding free space. The absolute length scale of the cavity drops out of $Q$ as per Eq. (1).

The folded chaotic whispering-gallery modes are surprisingly resilient even to large waveguide openings. For practical applications, much narrower waveguides will typically be desirable because the input and output should be single mode. We show results for large openings because that is the regime in which the coexistence of whispering-gallery modes with an apparent violation of Mather's theorem is clearest. In particular, the openings are much wider than the wavelength, so they cannot be treated as small perturbations. We also observe the non-monotonic distribution of $Q$-factors shown in Fig. 5 at $\epsilon=0.444$ for other deformations and waveguide widths. Figure 6 shows this for a higher deformation of $\epsilon=0.52$.

Figure 7 depicts the wave intensities of the highest-Q folded chaotic WGMs in Fig. 5, with wavenumbers ranging from $k \approx 13.6 \ldots 44$ at $n=2.4$. Each image represents a single quasibound state, not a superposition. The nodal structure that decorates the underly- 


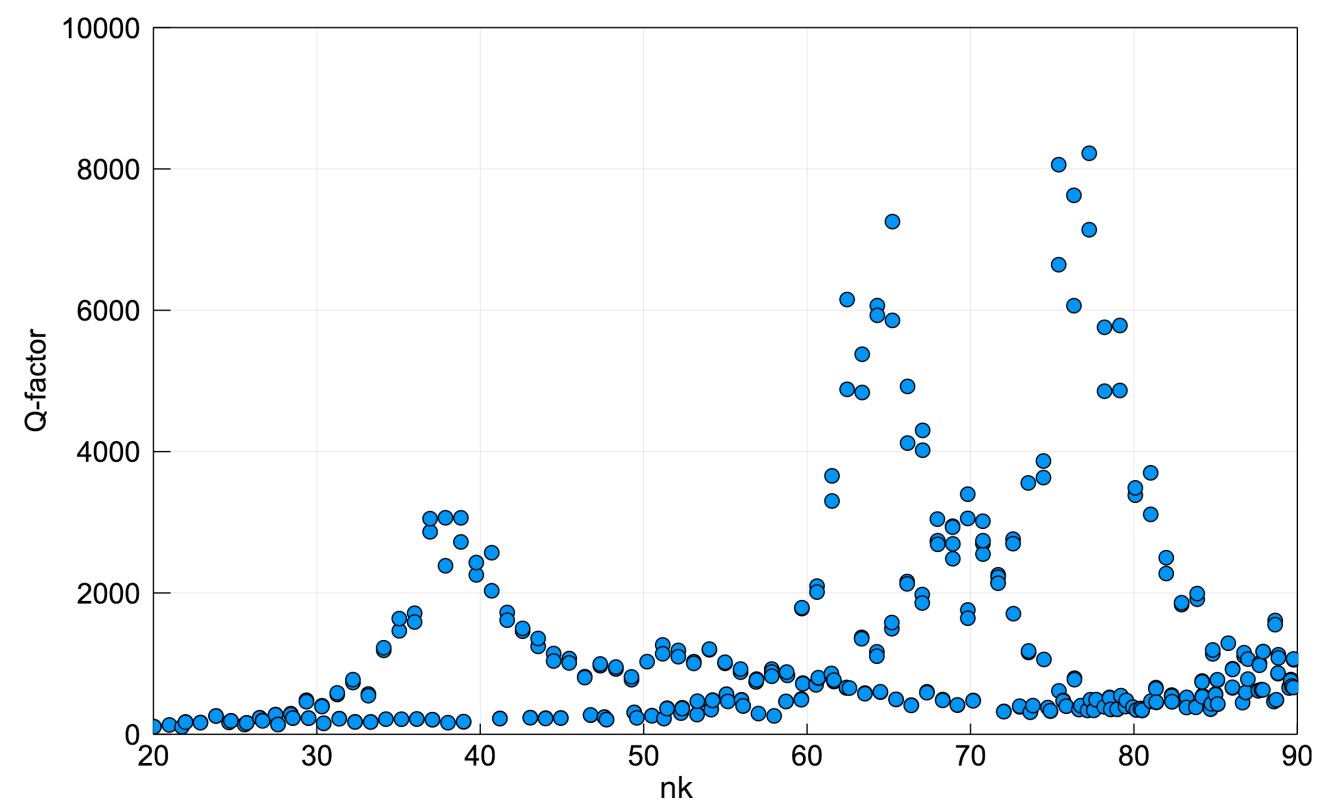

Figure 6. Q-factor scan versus interior wave number at deformation $\epsilon=0.52$ and refractive index $n=2.4$, showing modes with decay rate $\kappa<0.05$.

ing figure-eight patterns becomes more complex with increasing $k$, in much the same way that transverse nodes appear in higher-order Gaussian beams or radial nodes appear in whispering-gallery modes. The difference between those examples and the folded chaotic WGMs is that the nodal lines show wave dislocations typical of non-separable wave equations. Non-separability goes along with the chaotic ray dynamics of Fig. 3. Despite the increasingly complex nodal structure, all modes share the whispering-gallery like wave propagation along the convex parts of the boundary.

The Q-factor of any given mode may depend non-monotonically on the deformation parameter $\epsilon$ of Eq. (2), as shown in Fig. 1. However, we find other high-Q modes over the entire range of deformations in Fig. 1, as illustrated by the examples in Fig. 8. The robustness of the folded morphology against deformation is another interesting feature that these modes share with conventional whispering-gallery modes in convex resonators.

\section{B. Time-domain results}

To further validate the results of the boundary-integral method, we repeated the calculations for some of the modes using MEEP, which in particular allows for a different modeling of the waveguide attachments. Instead of assuming them to be finite stubs, we allowed them 

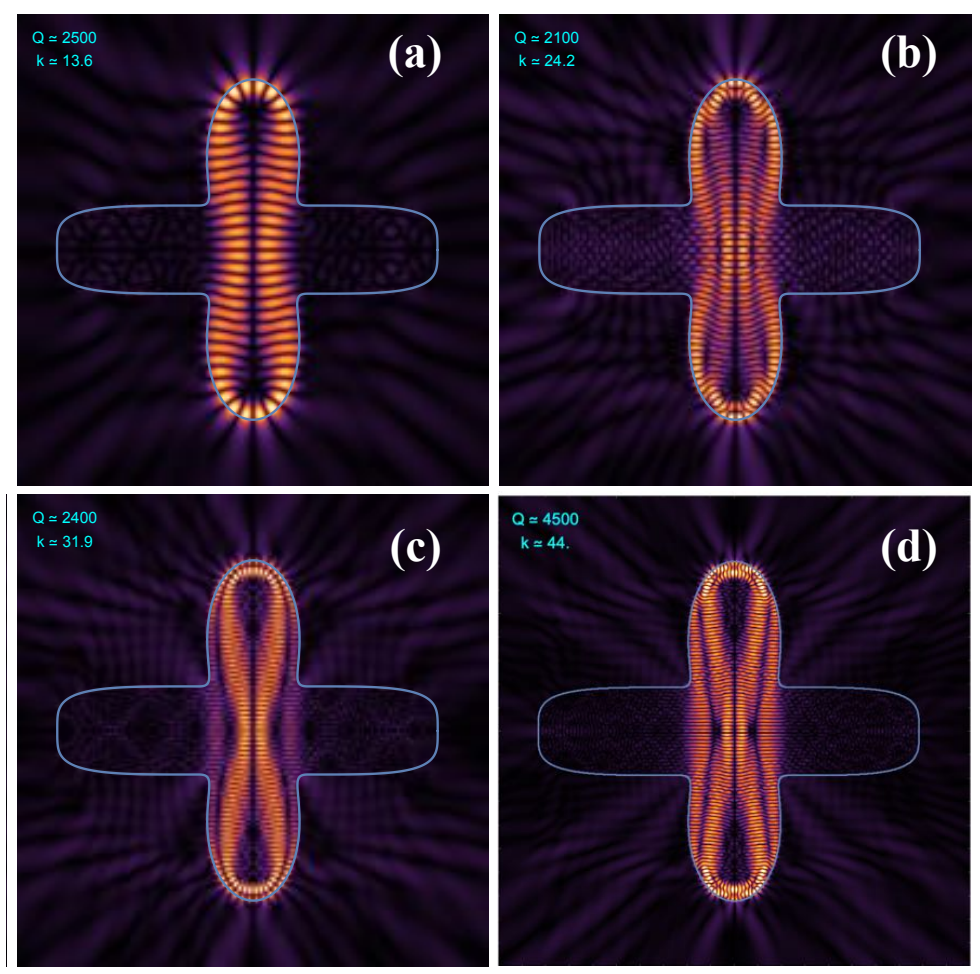

Figure 7. Field intensities for the modes corresponding to the first four Q-factor peaks in Fig. 5 for $n=2.4, \epsilon=0.444$. The Q factors are (a) 2500, (b) 2100, (c) 2400 and (d) 4500.

(a)

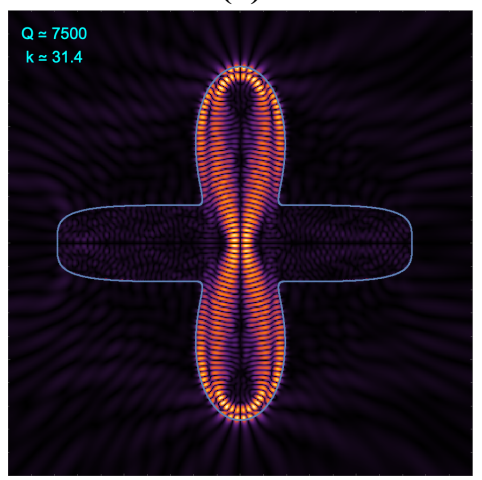

(b)

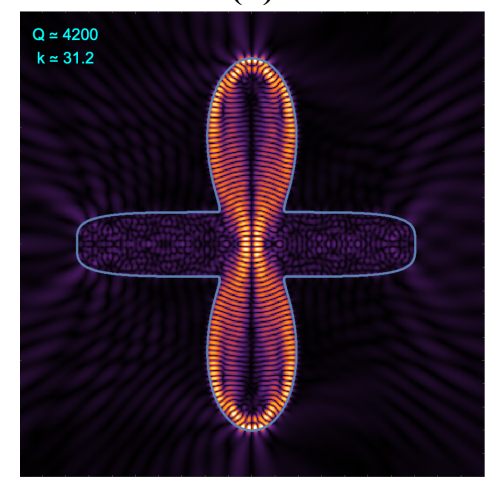

(c)

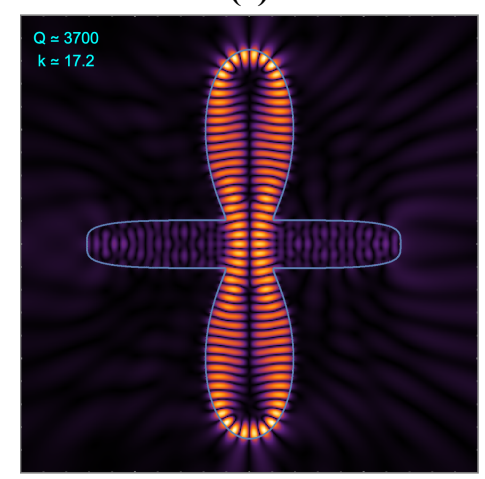

Figure 8. Field intensities for folded WGMs at different deformations: (a) $\epsilon=0.52$, (b) $\epsilon=0.6$, (c) $\epsilon=0.7$. The refractive index is $n=2.4$. In (c), a smaller wavenumber is chosen, whereas (a) and (b) have comparable wavenumbers. The narrowing of the horizontal stubs is a result of the requirement that its tangents must match the curve described by Eq. (2) at the corners.

to extend to the boundary of the simulation domain which includes a perfectly matched layer that prevents back-reflections of a wavepacket which was launched in one arm of the structure. After settling into a late-time decaying state, the wave field of a typical high-Q 

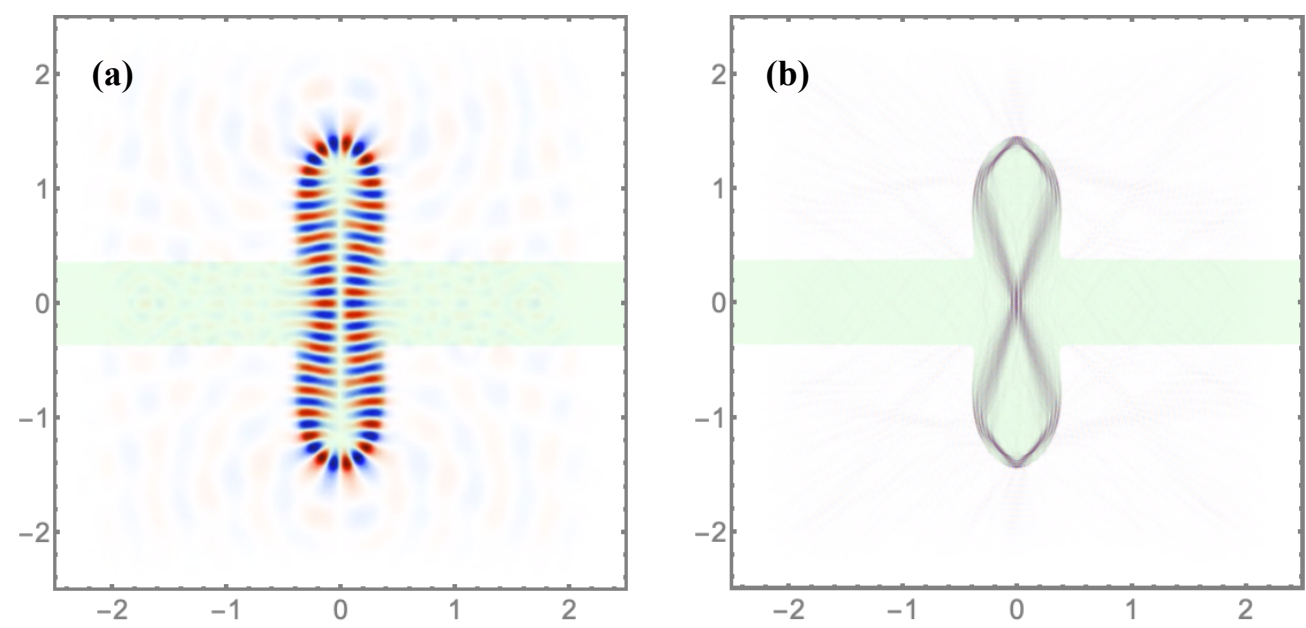

Figure 9. (a) Folded chaotic whispering-gallery mode as obtained in a finite-difference time domain computation for the same deformation $\epsilon=0.444$ and refractive index $n=2.4$ shown in Fig. 5 . The wave number corresponds to the top of the first $\mathrm{Q}$-factor peak, $k \approx 13.6$ ( $Q=2612)$. Waveguide and resonator structure are underlaid as green shading, and the false-color scale represents the electric field. (b) Same deformation and refractive index, but approximately ten times shorter wavelength, $k \approx 116.0$ and $Q=9898$. (b) is discussed in section IV.

mode displays the same pattern found in the previous section, cf. Fig. 9 (a). Shown in Fig. 9 (b) is the same resonator at a roughly tenfold shorter wavelength. The comparison illustrates why we make a distinction between folded chaotic WGMs as in Fig. 9 (a) and scarred states. The concentration of intensity on the six-bounce unstable periodic orbit of Fig. 2 (a) identifies the mode of Fig. 9 (b) as a scarred state, whereas there is no single periodic orbit that describes the intensity of a folded chaotic WGM.

Having convinced ourselves that the same results can be obtained with both numerical approaches (boundary-integral and finite-difference time domain), we proceed with MEEP to look for folded chaotic WGMs in resonators with thinner waveguides, cf. Fig. 10. The size of the opening in Fig. 10 (a) is comparable to the wavelength, whereas it is approximately twice the wavelength in (b). The whispering-gallery circulation along the boundary, characteristic of the earlier results in Fig. 1, shows that the phenomenon is robust not only to variations in the refractive index, but also to changes in waveguide width. 

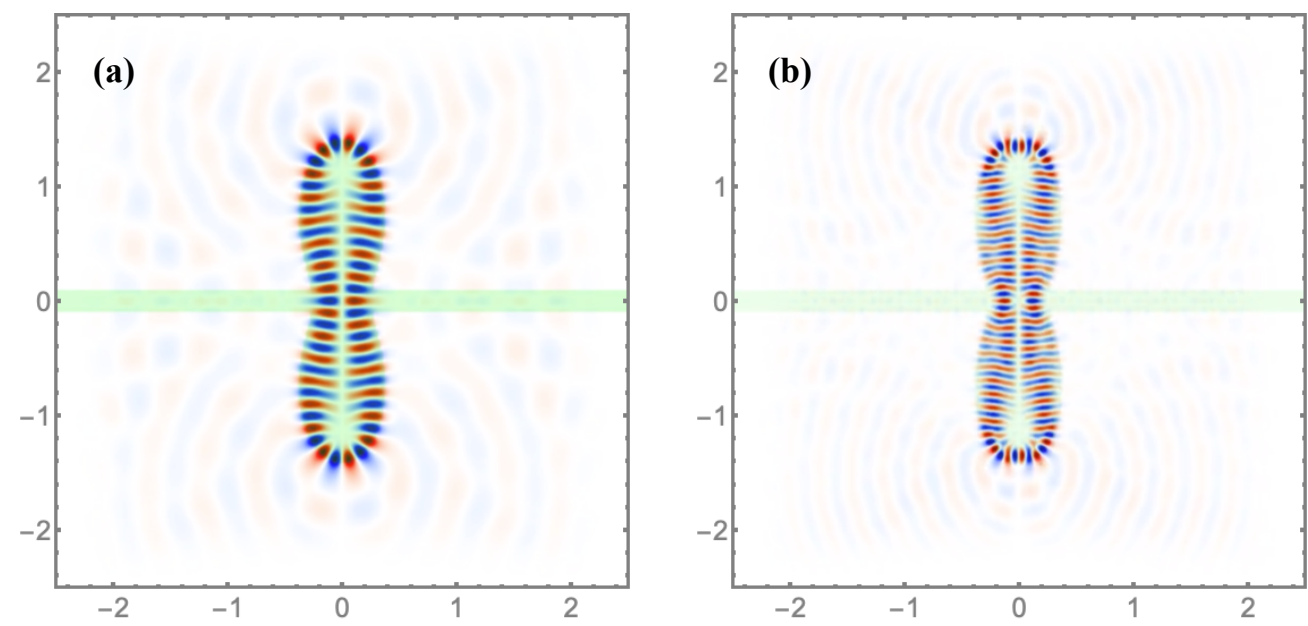

Figure 10. Two different modes with thinner waveguides, obtained by introducing a vertical offset between the two lobes of Eq. (2) and adjusting $\epsilon$ to match them smoothly to the horizontal waveguides. The refractive index is $n=2.4$. In (a) $k \approx 13.3$ and $Q \approx 1580$, in (b) $k \approx 22.4$ and $Q \approx 2550$.

\section{DISCUSSION}

\section{A. Folded chaotic whispering-gallery modes in phase space}

Individual modes of the folded chaotic WGM type do not shift or broaden significantly when the attached waveguides are shortened into stubs. From this we conclude that leakage into the waveguides is not the limiting factor for the lifetimes of individual folded chaotic WGMs. However, this is not straightforward to reconcile with the ray-tracing results depicted in Figs. 3 and 2 (e):

A comparison between the numerically observed peak Q factors in Fig. 5 and the classical ray escape into the waveguides provides a first indication that dynamical wave localization is essential in the formation of folded chaotic WGMs. We find that up to a set of measure zero (the trapped unstable periodic orbits), no matter where in the classical phase space we launch a ray, it escapes into the waveguide openings much too fast to explain the $Q$ factor found in the wave calculations.

The rapid ray escape can be viewed as a result of Mather's theorem, because the nonconvex billiard shape implies that the bounce dynamics does not constitute a twist map, and consequently neither the Lazutkin nor the Poincaré-Birkhoff theorems apply [4]. What remains is a largely chaotic phase space as in Fig. 3, and no stable ray orbits around which 
high-Q modes can be form by the mechanism of paraxial optics [22].

However, as suggested by Fig. 2 (e), even in a chaotic region of phase space, the motion is nevertheless organized by the periodic orbits: Each unstable periodic orbit is a periodic point of the Poincaré map which has stable and one unstable manifolds. In the linear regime near a periodic point, they correspond to trajectories that either converge on, or recede from, that point. By launching a large number of rays from a small neighborhood of the periodic point and iterating the billiard map forward and backward in time, these manifolds are traced out, revealing a characteristic web of intersections [31] - the homoclinic tangle. In the main panel of Fig. 11 (a), this has been done for the six-bounce orbit corresponding to the thick solid dots in Fig. 3. Embedded in the intersections between the manifolds are higher-order periodic points corresponding to orbits such as the one shown in Fig. 2 (c). Guided by the homoclinic tangle, the phase space flow is far from random on intermediate time scales and in fact helps explain the directional emission from free-standing asymmetric cavities [32] by predicting at what positions the condition for total internal reflection is first violated for a chaotically diffusing trajectory.

To expose the relationship between the observed modes and the underlying ray phase space structure exemplified in Fig. 3, it is useful to project the numerical wave results onto the phase space by means of the Husimi function. Choosing $\phi$ and $\sin \chi$ as the coordinates for this projection, only the boundary fields are needed, and this is just what the boundary-integral approach provides [33]. Therefore, Fig. 11 shows one of the high-Q modes as obtained with this method. In Fig. 5 it corresponds to the top of the peak structure located at $n k \approx 58$ as well as the mode depicted in Fig. 7 (b). The wave intensity shown in the inset illustrates the appearance of nodal lines which in a conventional WGM would be the radial zeros. As seen in Fig. 7, successive Q-factor peaks at higher $n k$ in Fig. 5 show additional such "radial" nodes, but the chaotic nature of the underlying phase space makes a rigorous classification in terms of radial nodal lines ambiguous.

The Husimi plot in Fig. 11 (b) instead classifies the mode according to the region of phase space by which it is supported - a procedure that is especially useful in this case because there are no stable ray orbits in the regions of the Poincare section bounded by the escape conditions. The six-bounce periodic orbit whose manifolds are explored in Fig. 11 (b) is also shown in Fig. 11 (b), colored yellow and red overlaying the Husimi intensity of the wave solution (the two colors belonging to the two opposite senses in which the figure-eight is 
(a)

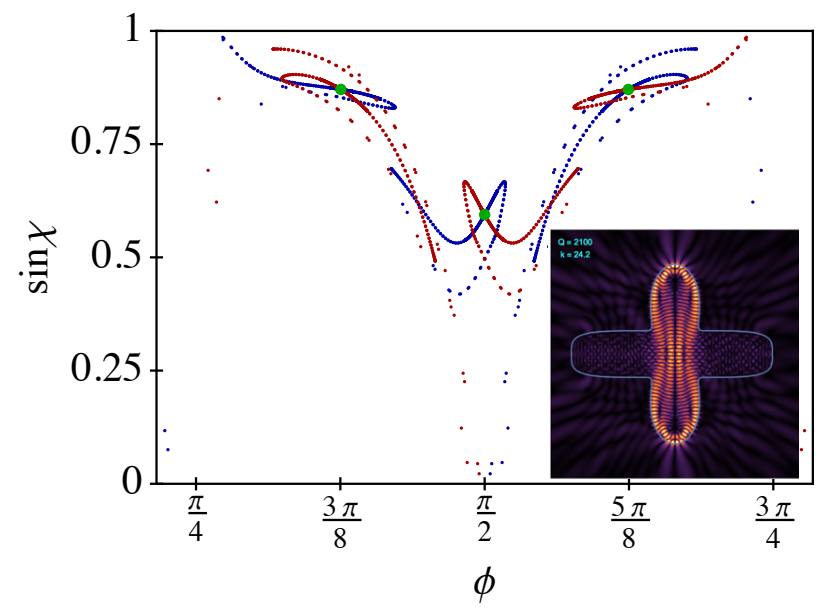

(b)

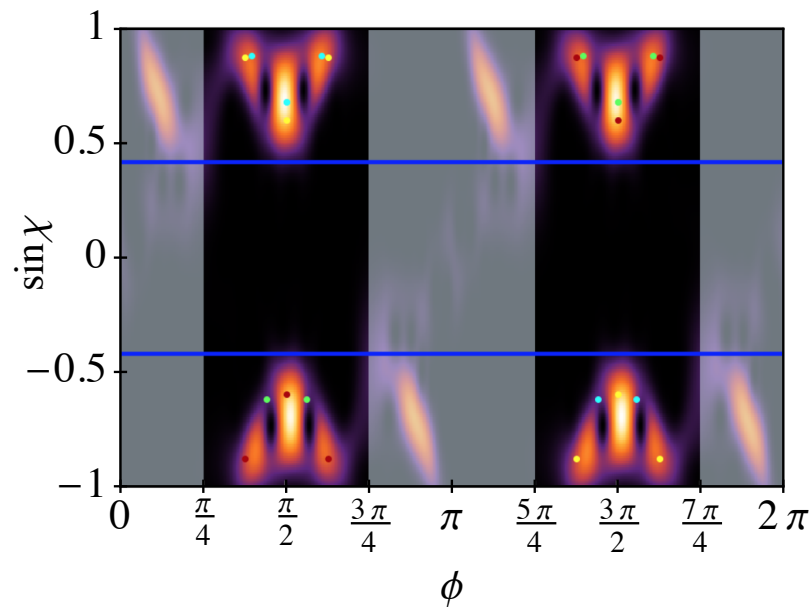

Figure 11. (a) Stable and unstable manifolds around an unstable periodic orbit. The zoomed-in region shown in (a) corresponds to the top left quadrant of the Husimi projection in (b). The realspace wave intensity of the mode in (b) is shown in the inset to (a). It is the same mode as shown in Fig. 7 (b). Shaded regions in (b) mark the waveguides, which were treated as escape windows in (a). The thin horizontal lines mark $\left|\sin \chi_{c}\right|=1 / 2.4$. Refractive escape is not considered in the ray simulation for (a), to get a more complete picture of the manifolds. Note the correspondence in shape between the phase space structure and the areas of large Husimi weight.

traversed). Shown in green and cyan are the self-intersecting period-five orbits. We have identified other periodic orbits with four to six bounces that are confined by total internal reflection in the same area of phase space, and it is not possible to uniquely assign regions of high Husimi intensity to a single orbit. However, Fig. 11 (b) does indicate unambiguously that the wave solution is in fact extended over a region of phase space bounded away from the critical angle $\chi_{c}$ by the V-shaped tangle of manifolds of Fig. 11 (a).

Figure 12 illustrates the common phase-space region shared between all high-Q modes. Just like the real-space wave functions, the Husimi intensity shows some variability between different modes. In particular, the areas of highest Husimi intensity are not centered on a single orbit of the many figure-eight type periodic orbits that exist in the cavity.

When interpreting the Husimi projection, the question arises whether our inability to assign a given mode to a unique unstable periodic orbit is merely a consequence of phasespace resolution. If this were true, it would mean the all the high-Q modes in our geometry 


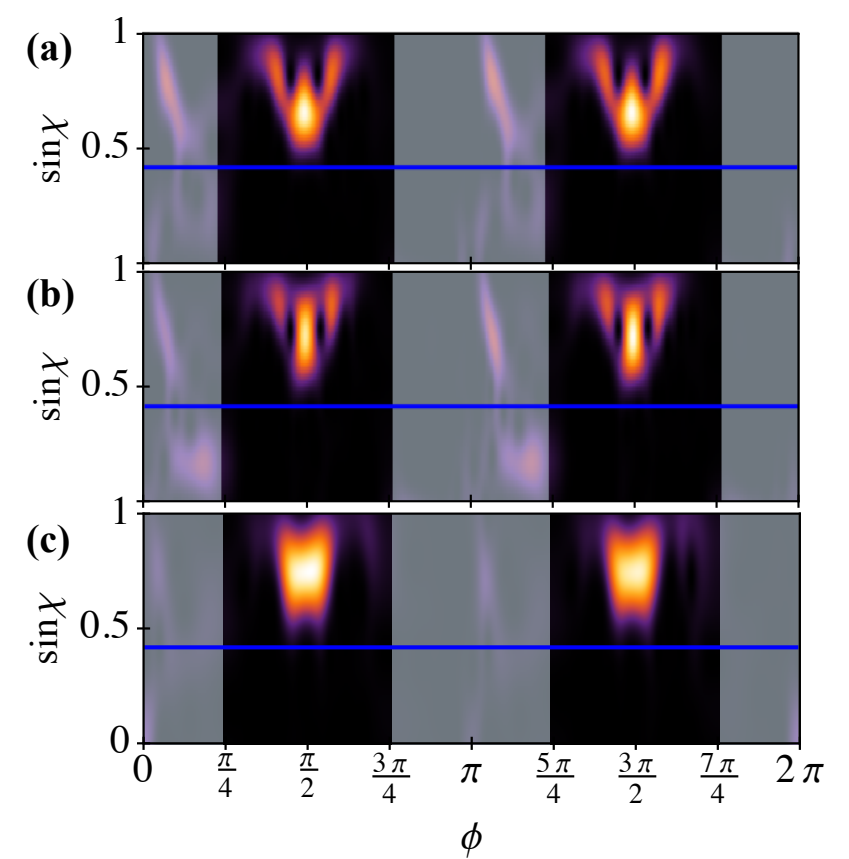

Figure 12. Comparison of Husimi plots for the same states shown in Fig. 8. Only the positive-sin $\chi$ half of the phase space is displayed (shaded regions and horizontal lines as in Fig. 11). The low wavenumber $(k \approx 17.2)$ in $(\mathrm{c})$ leads to lower phase-space resolution than in (a) and (b) where $k \approx 31.4$ and $k \approx 31.2$.

are really of the scarred kind shown in Fig. 9 (b). However, this is not the conclusion we draw. With the length scale used here, $k$ ranges from $k \approx 7$ in Fig. 4 to $k \approx 116$ in Fig. 9 (b). Within this approximate window, scarred modes belonging to a single unstable periodic orbit were not found below $k \approx 70$. The common feature of those lower- $k$ modes is instead the appearance of a caustic-like concentration of intensity following the curvature of the surface at $\phi \approx \pi / 2,3 \pi / 2$ (the top and bottom in the wave plots).

In a convex billiard, whispering-gallery circulation leads to true caustics, but there is also a dense set of periodic orbits in the shape of inscribed polygons with lengths that accumulate at the circumference of the billiard. This particular property of inscribed polygonal orbits in the whispering-gallery region of phase space finds its counterpart in our non-convex geometry: there is a similar accumulation of periodic orbits with figure-eight topology, characterized by an increasing number of bounces along either the top or the bottom portion of the billiard. The orbit displayed in Fig. 2 (c) is an example.

Two other possible scenarios are known to produce wave solutions localized on phase-space regions where the classical billiard shows no stable structure: marginally stable periodic 
orbits [1, 34], and ray orbits that become stabilized only due to the "softness" of the dielectric billiard boundary as a function of incident angle [35, 36]. In these cases, the wave intensity is "scar-like" in the sense that it again coalesces onto identifiable periodic orbits, which is not the case for the folded chaotic WGMs.

Given that purely ray based escape rates as shown in Fig. 2 (e) are too fast to explain the high Q factors, we therefore conclude that the folded chaotic WGMs are dynamically localized on the homoclinic tangle of the self-intersecting periodic orbits in this non-convex cavity.

\section{B. Q-factor oscillations}

The results shown in Figs. 5 and 6 are of particular practical importance: in designing resonators that exhibit modes with a folded topology, the geometric shape alone doesn't guarantee that $Q$ factors will increase monotonically with $k$, so the operating wavelength is constrained to match one of the $n k$ ranges where modes with high $Q$ can be found. Whispering-gallery modes of the perfect circle do not show any comparable grouping into peaks of higher and lower $Q$; instead, maximum attainable $Q$ factors grow monotonically with $n k$ because the modes with the lowest radial node number grow more and more concentrated at the surface, corresponding to grazing angle of incidence. Therefore, Figs. 5 and 6 show an aspect of the folded modes that distinguishes them from the conventional WGMs in convex resonators.

By varying the widths and lengths of the attached waveguides, we ruled out that the periodicity in $n k$ is due to threshold effects associated with the opening of new propagation channels. In an attempt to understand the $Q$-factor oscillations at the level of individual modes, we have singled out some high- $Q$ examples for closer investigation. In Fig. 5, the highest- $Q$ mode occurs at $n k \approx 105.6$. It is also shown in Fig. 7 (d) at $k \approx 44$. By following this mode over a neighborhood of deformation parameters $\epsilon$ around the shown values, we found that no crossings or anticrossings with other modes occur. Such anticrossings can in principle lead to non-monotonic $Q$-factors as a function of a system parameter, in particular when whispering-gallery and chaotic modes coexist because they often depend differently on said parameters [37].

Husimi plots provide phase-space information about the classical structures supporting 
the folded chaotic WGMs, but their resolution is limited by the Fourier uncertainty relation between position $\phi$ and angular momentum as measured by $\sin \chi$ [32]. Individual modes also vary in the relative weighting between the neighborhoods of the five- and six-bounce orbits, suggesting that an analysis of the Q-factor oscillations in Fig. 5 is best done not at the level of individual modes, but by adopting a more global point of view.

Such a global perspective can often be obtained by investigating the connection between the classical periodic orbits and the density of states [38]. In open systems, the density of states is continuous but can exhibit resonant structure because it is proportional to the Wigner-Smith delay time [32]. Oscillations in the number of high- $Q$ modes could be interpreted as oscillations in a density of states in which short-lived resonances are not counted. In order to relate these oscillations to periodic orbits, we explored the approach of reference [39], where beats between a small number of trajectories with similar actions in the periodic orbit sum were successfully used to interpret structure in the density of states for a Hénon-Heiles Hamiltonian. Using the period-orbit lengths $L_{1}$ and $L_{2}$ from Fig. 2 (a, b) to obtain the actions $S_{1,2}=n k L_{1,2}$, we arrive at an estimate for the beating period in Fig. 5 of

$$
\Delta(n k) \approx \frac{2 \pi}{\left|L_{1}-L_{2}\right|} \approx 30
$$

at refractive index $n=2.4$. Although this is in reasonable agreement with the spacing between the first two peaks in Fig. 5, we have not yet been able to reproduce the correct dependence of the numerically observed peak positions on deformation parameter $\epsilon$. In particular, we have found additional periodic orbits with larger values of $\left|L_{1}-L_{2}\right|$ that should appear with comparable amplitudes in the periodic-orbit sum for the density of states, so it remains to be seen if a refined version of Eq. (3) will be able to preserve the appealing simplicity of this interpretation in terms of periodic-orbit beats.

\section{CONCLUSIONS}

We have numerically calculated the quasi-bound state wave functions of a series of planar dielectric cavities with attached waveguides that support the formation of folded chaotic whispering-gallery modes. We considered a large range of wavelengths and refractive indices, using shape deformations that combine two convex halves in a non-focussing configuration. It is possible to sustain useful quality factors $\left(10^{3}\right.$ or larger $)$ despite the fact that the ray 
dynamics permits long-lived, trapped trajectories only for a discrete set of unstable periodic orbits. The figure-eight topology of these orbits imprints itself on the wave solutions even though there is no one-to-one correspondence between individual modes and single periodic orbits.

As a result of this folding, the modes are confined by total internal reflection in the uninterrupted convex parts of the resonator boundary, while at the same time exhibiting a waist that reduces their overlap with the opening to the attached waveguides. Because these openings are necessarily in violation of Mather's theorem, the existence of folded chaotic WGMs with Q-factors larger than $10^{3}$ is a wave localization effect. This localization is visualized with the help of Husimi projections which show that the long-lived modes are supported by a region of the ray phase space that coincides with the heteroclinic/homoclinic tangles of the unstable periodic orbits whose intensity pattern is also discernible in the real-space wave plots. In contrast to previous studies of dynamical localization in chaotic whispering-gallery like cavities, the angular momentum of the rays is not a slowly diffusing variable for our folded modes; it is the chaotic manifold structure that allows the wave modes to remain localized away from all escape windows in phase space.

As seen in the real-space plots, there are varying degrees of emission into the free space surrounding the structures. In this paper, the focus has been on the fact that high Q can be preserved in the presence of waveguide openings, because the latter are more intrusive than the curvature-induced violation of the total-internal reflection condition at the dielectric-air interface.

Having characterized the long-lived states for a range of deformations based on the resonator shape given in Eq. (2), explorations of modified shapes are currently in progress, with the additional goal of further elucidating the Q-factor peaks discussed in subsection IV B. By breaking the reflection symmetry of the cavity and going to higher wavenumbers in the wave simulations, we expect to improve upon the diagnostic value of the Husimi projections. We have already performed simulations for structures without spatial symmetries in order to make contact with previous work on unidirectional coupling [15], but additional work is needed to optimize the Q factors of those shapes.

A complementary approach to the quasibound-state analysis presented here is to investigate the resonances in transmission or reflection with the waveguides as input and output. In the stadium as the prototypical chaotic billiard, the transmission through attached leads 
has been studied in [40] in the context of electronic transport, i.e., with impenetrable walls. Sharp resonances are found to be associated with all regions of the chaotic phase space, leading to spectral statistics governed by level repulsion. In our system, the only long-lived modes are of the folded chaotic WGM type, and their groupings shown in Fig. 5 are not described by a universal random-matrix distribution. This goes hand in hand with the observation that no anti-crossings occur when varying the deformation parameter $\epsilon$ of Eq. (2), indicating that all modes respond to such variations in unison. A study of the transmission statistics for waveguide-coupled resonators of this type will provide insight into the interplay of the two types of escape windows in the phase space depicted in Fig. 3, where chaotic ray dynamics dominates similarly to the stadium, while at the same time the mode structure appears to be much simpler provided that low-Q states are discarded.

\section{ACKNOWLEDGMENT}

This work benefited from access to the University of Oregon high performance computer, Talapas.

\section{Appendix: Boundary Integral Implementation}

\section{Outline of the method}

To discover and investigate the resonant modes described, we developed software based on the boundary-integral approach as described in detail by Heider in Ref. [27]. The method is similar to that described in Ref. [41], which we have also applied to our system. The latter invariably produces spurious modes that make a large-scale analysis of the spectrum difficult, whereas the former does not produce any spurious modes at all (to our knowledge). This significant advantage can be traced back to the fact that Heider solves a set of simultaneous disretized of which one half differs from Ref. [41] in that it is obtained by taking an additional normal derivative of the integral equations for the fields in terms of their values derivatives on the dielectric interface. The price to be paid for this advantage is that great care must be taken in in numerically dealing with the singularities not just in the Green functions but also their derivatives. The following subsections address these details. 
Our software is written using the Julia programming language, which was chosen for its numeric performance, access to the necessary special-function libraries with implementations for complex arguments, and ease of integration with other numerical computing software. Our implementation is publicly available on GitHub [42]. It has been written for reusability and allows the user to specify their own boundary parameterizations. The implementation is written to utilize multiple processors on a single machine to parallelize operation where possible and has been tested on OS X and Linux.

To characterize the spectral patterns for folded chaotic WGMs, it was necessary to scan a wide range of $k$-space. Although the direct sweep method has the ability to find multiple resonances near an initial starting guess $k_{0}$, we observed that the ability to discern and identify relevant starting $k$ values for the secondary RII step decreases rapidly as $\left\|k_{0}-k\right\|$ increases. This is especially true for high-Q modes, as the resonance width for these modes are very narrow and we empirically observed that the direct sweep method discovers wider resonances in the vicinity of narrow resonances more easily which may mask the presence of the narrow resonances. Therefore, in order to reliably find the high-Q modes it was necessary to adopt a scanning procedure in which small regions of $k$-space were examined piecewise and candidate high-Q modes were identified within those small regions. We used the following algorithm:

1. Given a large $k$ range of interest from $k_{\min }$ to $k_{\max }$, choose a small step size $\left(k_{\text {step }}=.02\right)$ as a discretization.

2. Choose a target $\kappa$ for the imaginary part of $k$. We chose a small value $(\kappa=.0001)$ to target high-Q modes.

3. Starting at $k_{0}=k_{\min }-i \kappa$, execute the direct sweep procedure to generate candidate resonances.

4. Filter the candidate resonances to keep only those where $k_{0}-k_{\text {step }} \leq k_{\text {cand }} \leq k_{0}+k_{\text {step }}$, as they will be found again by a closer $k_{0}$ if outside that range and are less likely to converge to a valid resonance further away from $k_{0}$.

5. Increment $k_{0}$ by $k_{\text {step }}$ and repeat this procedure until the desired range has been covered. 
Because candidate $k$ values were retained both above and below the center point $k_{0}$, there was overlap between adjacent center points and most of the resonances which would eventually be discovered by the RII procedure would appear twice in the list of candidates. However, since different starting $k_{0}$ values were used in the direct sweep procedure, the exact values of these "duplicate" $k_{\text {cand }}$ were numerically slightly different as the candidates were discovered by a linear approximation to the eigenvalue problem around the vicinity of the initial guess $k_{0}$. In practice, when resolving the more accurate $k_{f}$ and the boundary field $x_{f}$ using the RII procedure, these slightly different starting points would converge to the same resonance within the numerical tolerances specified for the RII algorithm as well as display an identical spatial field pattern so we concluded that they were in fact the same mode. Therefore in this system it would have been possible to be more computationally efficient by combining or averaging very close candidate $k$ values found in adjacent regions of $k$-space spanned by the above algorithm, or in eliminating the overlap and keeping candidates at each step in the range $k_{0}-k_{\text {step }} / 2 \leq k_{\text {cand }} \leq k_{0}+k_{\text {step }} / 2$ or $k_{0} \leq k_{\text {cand }} \leq k_{0}+k_{\text {step }}$. We chose to err on the side of the extra computation in the interest of not missing any important resonances in the scan. It should be noted that there are other boundary geometries (such as the Reuleaux billiard, [33]) which exhibit very closely spaced doublets for which a careful approach may be necessary to avoid missing resonances.

Once the list of candidate $k_{0}$ values had been generated, the final $k_{f}$ and $x_{f}$ values were determined by first filtering the candidates to exclude very low-Q modes with $\kappa>1.0$ and then running the direct sweep procedure with starting guess $k_{0}=k_{\text {cand }}$ for each remaining candidate. The reason for the repeated direct sweep procedure was that since a large number of candidates were initially generated, we did not store the boundary fields $x$ associated with them. Since the input to the RII algorithm requires both the starting $k_{0}$ and the boundary field $x_{0}$, we needed to regenerate the boundary field. Since the initial candidates $k_{0}$ were already close to their final $k$, the subsequent direct sweep would in general produce inputs to the RII procedure that were even closer to the final convergent values and thus few iterations of the RII loop were required. 


\section{Some corrections to previous work}

We discovered two errors in the equations listed in the Appendix of Ref. [27]. The first appears in the description of $\tilde{N}$ below equation (A.6). There is a sign error in the last term. The corrected equation is:

$$
\begin{aligned}
\tilde{N}(t, \tau)= & \frac{i}{2} \bar{N}(t, \tau)\left\{\left(k n_{e}\right)^{2} H_{0}^{(1)}\left(k n_{e}|x(t)-x(\tau)|\right)-\frac{2 k n_{e} H_{1}^{(1)}\left(k n_{e}|x(t)-x(\tau)|\right)}{|x(t)-x(\tau)|}\right\} \\
& +\frac{i}{2} \frac{k n_{e} x^{\prime}(t) x^{\prime}(\tau)}{|x(t)-x(\tau)|} H_{1}^{(1)}\left(k n_{e}|x(t)-x(\tau)|\right) \\
& -\frac{i}{2} \bar{N}(t, \tau)\left\{\left(k n_{i}\right)^{2} H_{0}^{(1)}\left(k n_{i}|x(t)-x(\tau)|\right)-\frac{2 k n_{i} H_{1}^{(1)}\left(k n_{i}|x(t)-x(\tau)|\right)}{|x(t)-x(\tau)|}\right\} \\
& -\frac{i}{2} \frac{k n_{i} x^{\prime}(t) x^{\prime}(\tau)}{2|x(t)-x(\tau)|} H_{1}^{(1)}\left(k n_{i}|x(t)-x(\tau)|\right)
\end{aligned}
$$

The other error is in the equation for the diagonal terms $\tilde{N}_{2}(t, t)$, where the log terms should not use the norm of $x^{\prime}(t)$ squared, but simply the norm. The corrected equation is:

$$
\begin{aligned}
\tilde{N}_{2}(t, t) & =\frac{\left|x^{\prime}(t)\right|^{2}}{4 \pi}\left[\left(\left(k n_{e}\right)^{2}-\left(k n_{i}\right)^{2}\right)(\pi i-1-2 C)\right] \\
& +\frac{\left|x^{\prime}(t)\right|^{2}}{4 \pi}\left[-2\left(k n_{e}\right)^{2} \ln \left(\frac{k n_{e}\left|x^{\prime}(t)\right|}{2}\right)+2\left(k n_{i}\right)^{2} \ln \left(\frac{k n_{i}\left|x^{\prime}(t)\right|}{2}\right)\right]
\end{aligned}
$$

\section{Derivatives of the integral kernels}

Heider's boundary integral approach describes the $A$ matrix and its use in iteratively finding a resonant solution to the boundary integral equations. This iterative procedure requires the use of $A^{\prime}(k)$, both in the direct sweep procedure (Algorithm 3) and the Newton's

iteration step in the RII procedure (Algorithm 1). The derivation of the derivative matrix is straightforward and the results are outlined below, in terms of the constituent equations provided in the paper's appendix.

The $A^{\prime}(k)$ matrix is defined as

$$
A^{\prime}(k)=\left[\begin{array}{cc}
-\left(K_{e}^{\prime}(k)-K_{i}^{\prime}(k)\right) & -\left(S_{e}^{\prime}(k)-S_{i}^{\prime}(k)\right) \\
T_{e}^{\prime}(k)-T_{i}^{\prime}(k) & K_{e}^{* \prime}(k)-K_{i}^{* \prime}(k)
\end{array}\right]
$$


with the $K, S, T$, and $K^{*}$ integral operators being expressed after singularity subtraction in terms of equations

$$
\tilde{H}_{1}(k), \tilde{H}_{2}(k), \tilde{H}(k), \tilde{M}_{1}(k), \tilde{M}_{2}(k), \tilde{M}(k), \tilde{N}_{1}(k), \tilde{N}_{2}(k), \tilde{N}(k), \tilde{L}_{1}(k), \tilde{L}_{2}(k), \tilde{L}(k) .
$$

All other equations given in the appendix are independent of the wavenumber $k$.

First the $\tilde{H}$ equations:

$$
\begin{aligned}
\partial_{k} \tilde{H}_{1}(t, \tau, k) & =-\frac{1}{2 \pi} n(\tau)(x(t)-x(\tau))\left[k n_{e}^{2} J_{0}\left(k n_{e}|x(t)-x(\tau)|\right)-k n_{i}^{2} J_{0}\left(k n_{i}|x(t)-x(\tau)|\right)\right] . \\
\partial_{k} \tilde{H}_{1}(t, t, k) & =0 \\
\partial_{k} \tilde{H}_{2}(t, \tau, k) & =\partial_{k} \tilde{H}(t, \tau, k)-\partial_{k} \tilde{H}_{1}(t, \tau, k) \ln \left(4 \sin ^{2} \frac{t-\tau}{2}\right) \\
\partial_{k} \tilde{H}_{2}(t, \tau, k) & =\partial \tilde{H}(t, \tau, k)-\partial_{k} \tilde{H}_{1}(t, \tau, k) \ln \left(4 \sin ^{2} \frac{t-\tau}{2}\right) \\
\partial_{k} \tilde{H}_{2}(t, t, k) & =0 \\
\partial_{k} \tilde{H}(t, \tau, k) & =\frac{i}{2} n(\tau)(x(t)-x(\tau))\left[k n_{e}^{2} H_{0}^{(1)}\left(k n_{e}|x(t)-x(\tau)|\right)-k n_{i}^{2} H_{0}^{(1)}\left(k n_{i}|x(t)-x(\tau)|\right)\right]
\end{aligned}
$$

The $\tilde{H}_{1}^{*}, \tilde{H}_{2}^{*}$, and $\tilde{H}^{*}$, equations used in the starred operators $K^{*}$ are defined in terms of the unstarred equations with no additional $k$ dependence so their derivatives can use the results above.

The $\tilde{M}$ equations are:

$$
\begin{aligned}
& \partial_{k} \tilde{M}_{1}(t, \tau, k)=\frac{|x(t)-x(\tau)|}{2 \pi}\left[n_{e} J_{1}\left(k n_{e}|x(t)-x(\tau)|\right)-n_{i} J_{1}\left(k n_{i}|x(t)-x(\tau)|\right)\right] \\
& \partial_{k} \tilde{M}_{1}(t, t, k)=0 \\
& \partial_{k} \tilde{M}_{2}(t, \tau, k)=\partial_{k} \tilde{M}(t, \tau, k)-\partial_{k} \tilde{M}_{1}(t, \tau, k) \ln \left(4 \sin ^{2} \frac{t-\tau}{2}\right) \\
& \partial_{k} \tilde{M}_{2}(t, t, k)=0 \\
& \partial_{k} \tilde{M}(t, \tau, k)=-\frac{i|x(t)-x(\tau)|}{2}\left[n_{e} H_{1}^{(1)}\left(k n_{e}|x(t)-x(\tau)|\right)-n_{i} H_{1}^{(1)}\left(k n_{i}|x(t)-x(\tau)|\right)\right]
\end{aligned}
$$

The $\tilde{N}$ equations are: 


$$
\begin{aligned}
\partial_{k} \tilde{N}(t, \tau, k)= & -\frac{i}{2}|x(t)-x(\tau)| \bar{N}(t, \tau)\left[k n_{e}^{3} H_{1}^{(1)}\left(k n_{e}|x(t)-x(\tau)|\right)-k n_{i}^{3} H_{1}^{(1)}\left(k n_{i}|x(t)-x(\tau)|\right)\right] \\
& +\frac{i}{2} x^{\prime}(t) x^{\prime}(\tau)\left[k n_{e}^{2} H_{1}^{(1)}\left(k n_{e}|x(t)-x(\tau)|\right)-k n_{i}^{2} H_{1}^{(1)}\left(k n_{i}|x(t)-x(\tau)|\right)\right] \\
\partial_{k} \tilde{N}_{1}(t, \tau, k)= & \frac{1}{2 \pi}|x(t)-x(\tau)| \bar{N}(t, \tau)\left[k^{2} n_{e}^{3} J_{1}\left(k n_{e}|x(t)-x(\tau)|\right)-k^{2} n_{i}^{3} J_{1}\left(k n_{i}|x(t)-x(\tau)|\right)\right] \\
& -\frac{1}{2 \pi} x^{\prime}(t) x^{\prime}(\tau)\left[k n_{e}^{2} J_{0}\left(k n_{e}|x(t)-x(\tau)|\right)-k n_{i}^{2} J_{0}\left(k n_{i}|x(t)-x(\tau)|\right)\right] \\
\partial_{k} \tilde{N}_{1}(t, t, k)= & \frac{\left|x^{\prime}(t)\right|^{2} k\left(n_{i}^{2}-n_{e}^{2}\right)}{2 \pi} \\
\partial_{k} \tilde{N}_{2}(t, \tau, k)= & \partial_{k} \tilde{N}(t, \tau, k)-\partial_{k} \tilde{N}_{1}(t, \tau, k) \ln \left(4 \sin ^{2} \frac{t-\tau}{2}\right) \\
\partial_{k} \tilde{N}_{2}(t, t, k)= & \frac{\left|x^{\prime}(t)\right|^{2}}{2 \pi}\left[-2 k n_{e}^{2} \ln \left(\frac{k n_{e}\left|x^{\prime}(t)\right|}{2}\right)+2 k n_{i}^{2} \ln \left(\frac{k n_{i}\left|x^{\prime}(t)\right|}{2}\right)-k\left(n_{e}^{2}-n_{i}^{2}\right)(2+2 C-\pi i)\right]
\end{aligned}
$$

As in the reference, $C \approx 0.5772156649$ refers to the Euler-Mascheroni constant.

Finally, the $\tilde{L}$ equations:

$$
\begin{aligned}
\partial_{k} \tilde{L}_{1}(t, \tau, k)= & \frac{|x(t)-x(\tau)|}{2 \pi}\left[\left(k^{2} n_{e}^{3} J_{1}\left(k n_{e}|x(t)-x(\tau)|\right)-k^{2} n_{i}^{3} J_{1}\left(k n_{i}|x(t)-x(\tau)|\right)\right)\right] \\
& \left.\left.-\frac{1}{\pi}\left[k n_{e}^{2} J_{0}\left(k n_{e}|x(t)-x(\tau)|\right)\right)-k n_{i}^{2} J_{0}\left(k n_{i}|x(t)-x(\tau)|\right)\right)\right] \\
\partial_{k} \tilde{L}_{1}(t, t, k)= & \frac{k\left(n_{i}^{2}-n_{e}^{2}\right)}{\pi} \\
\partial_{k} \tilde{L}_{2}(t, \tau, k)= & \partial_{k} \tilde{L}(t, \tau, k)-\partial_{k} \tilde{L}_{1}(t, \tau, k) \ln \left(4 \sin ^{2} \frac{t-\tau}{2}\right) \\
\partial_{k} \tilde{L}_{2}(t, t, k)= & -\frac{1}{\pi}\left[2 k n_{e}^{2} \ln \left(k n_{e}\right)-2 k n_{i}^{2} \ln \left(k n_{i}\right)+k\left(n_{e}^{2}-n_{i}^{2}\right)\left(2 \ln \left(\frac{\left|x^{\prime}(t)\right|}{2}\right)-\pi i+2 C+1\right)\right] \\
\partial_{k} \tilde{L}(t, \tau, k)= & -\frac{i}{2}|x(t)-x(\tau)|\left[k^{2} n_{e}^{3} H_{1}^{(1)}\left(k n_{e}|x(t)-x(\tau)|\right)-k^{2} n_{i}^{3} H_{1}^{(1)}\left(k n_{i}|x(t)-x(\tau)|\right)\right] \\
& +i\left[k n_{e}^{2} H_{0}^{(1)}\left(k n_{e}|x(t)-x(\tau)|\right)-k n_{i}^{2} H_{0}^{(1)}\left(k n_{i}|x(t)-x(\tau)|\right)\right]
\end{aligned}
$$

It is hoped that the explicit formulas given here provide a complete picture of the computational method we employed in finding the quasibound states.

[1] H. Cao and J. Wiersig, Reviews of Modern Physics 87, 61 (2015).

[2] J. U. Nöckel and A. D. Stone, Nature 385, 45 (1997).

[3] Z.-P. Liu, X.-F. Jiang, Y. Li, Y.-F. Xiao, L. Wang, J.-L. Ren, S.-J. Zhang, H. Yang, and Q. Gong, Applied Physics Letters 102, 221108 (2013). 
[4] V. F. Lazutkin, KAM Theory and Semiclassical Approximations to Eigenfunctions (Springer, New York, 1993).

[5] C. D. Schwieters, J. A. Alford, and J. B. Delos, Phys. Rev. B 54, 10652 (1996).

[6] J. N. Mather, Ergodic Theory and Dynamical Systems 4, 301 (1984).

[7] L. A. Bunimovich, Commun. Math. Phys. 65, 295 (1979).

[8] J. U. Nöckel, A. D. Stone, and R. K. Chang, Opt. Lett. 19, 1693 (1994).

[9] S. A. Backes, J. R. A. Cleaver, A. P. Heberle, and K. Köhler, J. Vac. Sci. Technol. B 16, 3817 (1998).

[10] X.-M. Lv, Y.-Z. Huang, Y.-D. Yang, H. Long, L.-X. Zou, Q.-F. Yao, X. Jin, J.-L. Xiao, and Y. Du, Opt. Express 21, 16069 (2013).

[11] B.-J. Li and P.-L. Liu, IEEE J. Quantum Electron. 33, 1489 (1997).

[12] C. Yan, J. Shi, P. Li, H. Li, and J. Zhang, Opt. Laser Technol. 56, 285 (2014).

[13] Q. Song, L. Ge, B. Redding, and H. Cao, Phys. Rev. Lett. 108, 243902 (2012).

[14] S. Liu, W. Sun, Y. Wang, X. Yu, K. Xu, Y. Huang, S. Xiao, and Q. Song, Optica 5, 612 (2018).

[15] Y.-D. Yang, Y. Zhang, Y.-Z. Huang, and A. W. Poon, Opt. Express 22, 824 (2014).

[16] K.-J. Che, J.-D. Lin, Y.-Z. Huang, Y.-D. Yang, J.-L. Xiao, and Y. Du, IEEE Photon. Technol. Lett. 22, 1370 (2010).

[17] Y.-Z. Huang, Y.-D. Yang, S.-J. Wang, J.-L. Xiao, K.-J. Che, and Y. Du, Sci. China Ser. E: Technol. Sci. 52, 3447 (2009).

[18] L. Zou, X. Lv, Y. Huang, H. Long, J. Xiao, Q. Yao, J. Lin, and Y. Du, IEEE J. Sel. Top. in Quantum Electron. 19, 1501808 (2013).

[19] T. Fukushima, T. Tanaka, and T. Harayama, Opt. Lett. 32, 3397 (2007).

[20] R. E. Prange, R. Narevich, and O. Zaitsev, Phys. Scr. T90, 134 (2001).

[21] C. Gmachl, F. Capasso, E. E. Narimanov, J. U. Nöckel, A. D. Stone, J. Faist, D. L. Sivco, and A. Y. Cho, Science 280, 1556 (1998).

[22] V. M. Babic and V. S. Buldyrev, Short-wavelength diffraction theory (Springer Verlag, Berlin, 1972).

[23] W. Fang, H. Cao, V. A. Podolskiy, E. E. Narimanov, Opt. Express 13, 5641 (2005).

[24] J. U. Nöckel, Phys. Scr T90, 263 (2001).

[25] F. Lackner, I. Brezinova, J. Burgdörfer, and F. Libisch, Phys. Rev. E 88, 022916 (2013). 
[26] M. Fernández Guasti, Int. J. Math. Educ. Sci. Technol. 23, 895 (1992).

[27] P. Heider, Comput. Math. Appl. 60, 1620 (2010).

[28] J. U. Nöckel and A. D. Stone, in Optical Processes in Microcavities, edited by R. K. Chang and A. J. Campillo (World Scientific, Singapore, 1996).

[29] V. A. Mandelshtam, Prog. Nucl. Magn. Reson. Spectrosc. 38, 159 (2001).

[30] A. F. Oskooi, D. Roundy, M. Ibanescu, P. Bermel, J. D. Joannopoulos, and S. G. Johnson, Comput. Phys. Commun. 181, 687 (2010).

[31] A. J. Lichtenberg and M. A. Lieberman, Regular and Chaotic Dynamics, Second Edition (Springer-Verlag, New York, 1992).

[32] J. U. Nöckel and R. K. Chang, in Cavity-Enhanced Spectroscopies, edited by R. D. van Zee and J. P. Looney (Academic Press, San Diego, 2002), vol. 40 of Experimental Methods in the Physical Sciences, pp. 185-226.

[33] B. Dietz, T. Guhr, B. Gutkin, M. Miski-Oglu, and A. Richter, Phys. Rev. E 90, 022903 (2014).

[34] J. Wiersig, Phys. Rev. Lett. 97, 253901 (2006).

[35] D. H. Foster, A. K. Cook, and J. U. Nöckel, Opt. Lett. 32, 1764 (2007).

[36] J. Unterhinninghofen, J. Wiersig, and M. Hentschel, Phys. Rev. E 78, 016201 (2008).

[37] G. Hackenbroich and J. U. Nöckel, Europhys. Lett. 39, 371 (1997).

[38] M. Brack and R. K. Bhaduri, Semiclassical Physics (Westview Press, Boulder, Co., 2003).

[39] M. Brack, R. K. Bhaduri, J. Law, and M. V. N. Murthy, Phys. Rev. Lett. 70, 568 (1993).

[40] H. Ishio, A. I. Saichev, A. F. Sadreev, and K.-F. Berggren, Comput. Phys. Commun. 142, 64 (2001).

[41] J. Wiersig, J. Opt. A: Pure Appl. Opt. 5, 53 (2003).

[42] https://github.com/kahliburke/BoundaryIntegralMethod.jl 